\title{
Epidemiological and clinical characteristics of human brucellosis in Razavi Khorasan proivince and important risk factors in the years 2008 to 2013
}

\author{
Type of article: conference abstract \\ Soheil Hashtarkhani1, ${ }^{*}$ \\ 1: Ph.D student, Department of medical informatics, Faculty of Medicine, University of \\ Mashhad Medical Science, Mashhad, Iran. \\ * hashtarkhanis951@mums.ac.ir
}

\begin{abstract}
Background \& Purpose: Brucellosis is a transmissible disease between humans and animals that is transmitted through infected animals and their products. Razavi Khorasan which is classified as a high incidence province, considered as one of the notable areas for controlling the disease.

Materials \&Methods: Data on 5743 cases in Razavi Khorasan (Mashhad Medical University Coverage) over 5 years were analyzed.A GIS map is designed to show and compare incidence rate among different regions using ArcGIS software and different correlations between patients specifications are analysed.

Results: The mean incidence of disease in the province is 26 per hundred thousand people which is highly correlated to animalpopulation of every area. In the first 3 years of study the disease incidence was ascending and in last 2 years it was descending and in May till August the prevalence was more than other months.85\% of patients were rural and $15 \%$ were urban. $56.9 \%$ were male and $43.1 \%$ female. Household and farmer-rancher were the most common jobs. Average age of patients was $33.4 \pm 18.1$ year. $77.2 \%$ had consumption of unpasteurized dairy products which milk (91.4\%) and cheese (21.4\%) had the highest consumption. The most common symptoms were fever (79\%) and anorexia (47.5\%). A significant relationship between location and both livestock vaccination history and also family history, was found.

Conclusion: because of higher incidence rate of the disease in Daregaz,Takhtjolge and Rashtkhar, and young people aged 10 to 30 years and villagers, It's need to control the disease in these groups.

Keywords: Brucellosis, Epidemiology, Razavi Khorasan.
\end{abstract}

\section{Declaration of conflicts}

This abstract is selected from the First International Congress of Diseases and Health Outcomes Registry and First National Congress of Medical Informatics, 14-17 February 2017, Mashhad, Iran

\section{Authors' biography}

No biography.

\section{References}

No references. 